\title{
Research on monocrystalline silicon defect detection based on wavelet singularity
}

\author{
Wu Wei ${ }^{1, a^{*}}$, Qiu Zongming ${ }^{2, b}$, Huang Qiu hong ${ }^{3, c}$ \\ 1.Xi'an University of Technology, School of Mechanical and Precision Instrument Engineering, Xi'an ,710048, China \\ 2.Xi'an University of Technology, School of Mechanical and Precision Instrument Engineering, Xi'an ,710048, China \\ 3.Xi'an University of Technology, School of Mechanical and Precision Instrument Engineering, Xi'an ,710048, China \\ a402374021@qq.com, b2218397083@qq.com, ${ }^{\circ 619817646 @ q q . c o m ~}$
}

Keywords:monocrystalline silicon; defect; wavelet transform; Lipschitz exponent

\begin{abstract}
In order to improve the process quality of monocrystalline silicon slicing, the analysis method based on the wavelet transform and the Lipschitz exponent was employed to explore the local singularity of defect detection signal for monocrystalline silicon through the self-built ultrasonic traversal detection platform. It was convenient to obtain the defect data and to complete a three-dimensional reconstruction of defects. Besides, the feasibility and availability of the method were validated by comparing the tested values which were obtained through the analysis of noise reduction of flaw signal using multiple wavelet bases. Experimental results reveal that the method can greatly improve the efficiency of slicing processes and the relative error is less than $4 \%$. Meanwhile, this method can be widely used for the extraction of the ultrasonic signal eigenvalue and fault detection etc. with little computational complexity.
\end{abstract}

\section{Introduction}

During the preparation of monocrystalline silicon, some defects, such as bubbles, inclusion etc. in silicon rods are formed due to the effects of many factors. These defects have a negative effect on the future process and the quality of slicing. Therefore, to guarantee the quality of the finished products and improve working efficiency as well as equipment protection, cost reduction etc., researches on monocrystalline silicon materials primarily require to be performed on nondestructively finding out the existence of defects in silicon rods in advance and effectively conducting their localization and quantitative analysis. In the course of the engineering detection, the positions and sizes of defects can be obtained through the information of ultrasonic flaw echo signal. However, the electrical noise caused by ultrasonic detector and the background noise [1][9][10] brought by the internal scattering center of material usually disturb the detection of defect location 
even overwhelm the original flaw signals [2]. It presents great challenge to further data analysis. Therefore, it is essential to conduct the research on the method of effective defect location.

Based on the self-built circumference traversal testing platform, the relationship between the signal singularity and wavelet transform, and the theory and method of accurately reconstructing the original signal by the maximum modulus of wavelet transform were applied in this research. Moreover, to locate the accurate positions of flaw ultrasonic signal in monocrystalline silicon, the decrement of the local maximum modulus of wavelet transform was utilized to obtain Lipschitz exponent with the extreme points and zero-crossing points using Mallat. Experimental results prove that the features of wavelet transform and Lipschitz exponent can precisely reflect the abrupt change of defect wave of monocrystalline silicon. They also prove the availability of algorithms.[3 5]

\section{Mathematical model of ultrasonic echo}

In the wideband ultrasonic detection, the ultrasonic echo signal commonly is a wideband signal modulated by the center frequency of probe. The mathematical model of ultrasonic echo can be established as follow [6]:

$$
\mathrm{f}(\mathrm{t})=\mathrm{h}(\mathrm{t}) \cos \left(2 \mathrm{Pf}_{0} \mathrm{t}+\mathrm{U}\right)
$$

where $\mathrm{f}_{0}$ stands for the center frequency of probe; the bandwidth of $\mathrm{f}(\mathrm{t})$ is determined by $\mathrm{B}_{0}$; the power spectrum of ultrasonic pulse signal is usually modeled as Gaussian function [7]. When envelope $h(t)$ is considered as Gaussian function, Formula 1 turns to be:

$$
f(t)=\frac{\exp \left(-t^{2} / 4 B_{0}\right)}{4 P B_{0}} \operatorname{COS}\left(2 P f_{0} t+U\right)
$$

It means that the mathematical model of ultrasonic flaw echo is modulated from Gaussian function. If $\mathrm{L}(\mathrm{f}) \exp (\mathrm{iH}(\mathrm{f})$ ) is the frequency domain modeling of noise $\mathrm{n}(\mathrm{t})$, represented by $\mathrm{N}$ ( f ), the formula for the signal frequency domain accepted by system will be:

$$
C(f)=2 A H(f)\left|\frac{A t_{c}}{T} \frac{\sin \left(P f t_{c}\right)}{P f t_{c}}\right| \sum_{n=-\infty}^{+\infty} D \hat{u} f-f_{0}-n \frac{1}{T}+L(f) \exp (i H(f))
$$

Most often, Lipschitz exponent is employed to describe the local singularity of function. The definition is followed.

Definition 1 [8] Suppose $\mathrm{n}$ is a nonnegative integer, $\mathrm{n}<\mathrm{a} \leqslant \mathrm{n}+1$, if constants $A$ and $\mathrm{h}_{0}>0$, and the polynomial of degree $\mathrm{n}$, i.e. $\mathrm{P}_{\mathrm{n}}(\mathrm{h})$, can make any $\mathrm{h} \leqslant \mathrm{h}_{0}$, all can be

$$
\left|f\left(x_{0}+h\right)-P_{n}(h)\right| \leq A|h|^{a}
$$

In the situation, $\mathrm{f}(\mathrm{x})$ at point $\mathrm{x}_{0}$ is called as Lipschitz $\alpha$.

The larger the Lipschitz exponent, the smoother the function. When the function is continuous and differentiable at a point, the Lipschitz exponent will be 1 there. If at a point it is derivable and the derivate is bounded but not continuous, the Lipschitz exponent is still 1, while if the Lipschitz exponent of $\mathrm{f}(\mathrm{x})$ is less than 1 at point $\mathrm{x}_{0}$, the function is singular at the point. Moreover, if the function is discontinuous but bounded at point $\mathrm{x}_{0}$, the Lipschitz exponent is 0 . 


\section{Singularity detection of signals based on wavelet transform ${ }^{[7]}$}

The traditional method to analyze the singularity of function $f(x)$ is to investigate the asymptotic decay of Fourier transform $f(\omega)$ for $f(x)$. If a bounded function $f(x)$ is a coincident Lipschitz exponent which can meet the formula following:

$$
\int_{-\infty}^{\infty}|f(\omega)|(1+|\omega|) d \omega<+\infty
$$

However, Formula 5 is a sufficient condition so that it can provide the singularity metrics of $\mathrm{f}(\mathrm{x})$ in universe but cannot be applied to research the local singularity of a specific point $\mathrm{x}_{0}$. But the local singularity can be analyzed by wavelet. The value of wavelet coefficient depends on the property of $\mathrm{f}(\mathrm{x})$ in the neighborhood of $\mathrm{x}_{0}$ and the scale used by wavelet transform. At the smaller scale, it can provide the local property of $\mathrm{f}(\mathrm{x})$. Through theorems 1 and 2 have explained the relationship between asymptotic decay and local Lipschitz property of wavelet transform coefficient at the small scale. In this paper, wavelet transform is represented by $\mathrm{Wf}(\mathrm{s}, \mathrm{x})$ and $\mathrm{s}$ means scale in function $\mathrm{f}(\mathrm{x})$.

Theorem 1: Suppose $\mathrm{f}(\mathrm{x}) \in \mathrm{L} 2(\mathrm{R}),[\mathrm{a}, \mathrm{b}]$ is a closed interval in $\mathrm{R}$, and $0<\mathrm{a}<1$. If $\forall \varepsilon>0$, $f(x)$ is a function when the coincident Lipschitz exponent is a at $(a+\varepsilon, b-\varepsilon)$. Only if there is a constant $A \varepsilon$ and $x \in(a+\varepsilon, b-\varepsilon)$, for any $s>0$ can be

$$
|W f(s, x)| \leq A_{\varepsilon} s^{a}
$$

Relative to Formula (5), Theorem 1 can provide the local asymptotic decay rather than the global one. In order to make sure Theorem 1 can be used, when the Lipschitz exponent is more than one, suppose the wavelet $\varphi(\mathrm{x})$ has vanishing moment with enough orders, which can be called as wavelet $\varphi(\mathrm{x})$ with $\mathrm{n}$ vanishing moments. If all positive integer $\mathrm{k}<\mathrm{n}$, we obtain

$$
\int_{-\infty}^{\infty} x^{k \varphi}(x) d x=0
$$

If the wavelet $\varphi(\mathrm{x})$ has $\mathrm{n}$ vanishing moments, Theorem 1 is still valid for any non-integer $(0<\mathrm{a}<\mathrm{n})$, while it may not fit the Lipschitz exponent for integer (Formula 6). In addition, Theorem 1 just provides the Lipschitz regularity metrics in the interval.

Theorem 2: Set $\mathrm{n}$ is a positive integer and $\mathrm{a}<\mathrm{n}, \mathrm{f}(\mathrm{x}) \in \mathrm{L}^{2}(\mathrm{R})$. If at the point $\mathrm{x}_{0} \mathrm{f}(\mathrm{x})$ is Lipschitz exponent, a, there will be a constant $A$ to make all $\mathrm{x}$ in the neighborhood of $\mathrm{x}_{0}$ and any scale $\mathrm{s}>0$ meeting

$$
|W f(s, x)| \leq A\left(s^{a}+\left|x-x_{0}\right|\right)
$$

On the contrary, if a is non-integer and less than $n$, function $f(x)$ is Lipschitz exponent a at the point of $\mathrm{x}_{0}$ when the two conditions below are true.

(1) A certain $\varepsilon>0$ and constant $A$, for any point $x$ and any scale $s$ in the neighborhood of $x_{0}$ can be:

$$
|W f(s, x)| \leq A s^{\varepsilon}
$$

there is a constant $\mathrm{B}$ to make any point $\mathrm{x}$ and any scale $\mathrm{s}$ in the neighborhood of $\mathrm{x}_{0}$ can be: 


$$
|W f(s, x)| \leq B\left[s^{a}+\frac{\left|x-x_{0}\right|}{|\log | x-x_{0}||}\right]
$$

Form Theorem 1, it can be known that Formula (9) means that $\mathrm{f}(\mathrm{x})$ is consistent Lipschitz exponent $\varepsilon$ which can be arbitrarily small when $\mathrm{x}_{0}$ is in a certain neighborhood.

In order to explain Formulas (8) and (10),

At first to define the cone in scale space, it needs to meet the condition:

$$
\left|x-x_{0}\right| \leq s
$$

For the point $(\mathrm{s}, \mathrm{x})$ in the cone, Formulas (8) and (10) indicate that, when $\mathrm{s}$ tends to be 0 , $|W f(s, x)|=O\left(s^{a}\right)$. The argument of $|W f(s, x)|$ below the cone relies on the a-th power of the distance between $\mathrm{x}$ and $\mathrm{x}_{0}$. However, the essential condition and the sufficient condition have different upper bounds. Formula (10) shows that the point $(\mathrm{s}, \mathrm{x})$ below the cone is

$$
|W f(s, x)|=O\left(\left(\left|x-x_{0}\right|^{a}\right) /\left(\log \left|x-x_{0}\right|\right)\right) \text {. The behavior of wavelet transform in the cone points to } \mathrm{x}_{0} .
$$

Commonly, the two components below the cone needs to be conducted respectively.

The theorems mentioned demonstrate that the wavelet transform can be utilized to estimate the local singularity of function. But in most cases, the conclusion is difficult to directly detect the local singularity of function and to estimate Lipschitz exponent. Since the arithmetic labor is larger, using the relationship between the local maximum of wavelet transform and the singular point of function for analysis can reduce the arithmetic labor greatly.

\section{Singularity detection and the maximum modulus of wavelet transform [10]}

Definition 2 [8] at the scale of $\mathrm{s} 0$, point $(\mathrm{s} 0, \mathrm{x} 0)$ is regarded as the local maximum point, if

$$
\frac{\partial W f\left(s_{0}, x\right)}{\partial x}
$$

when $\mathrm{x}=\mathrm{x} 0$, there is a zero-crossing point, point $(\mathrm{s} 0, \mathrm{x} 0)$ can be called as the maximum modulus point of wavelet transform. For arbitrary point $\mathrm{x}$ in a certain neighborhood of $\mathrm{x} 0$, we have $\mid \mathrm{Wf}(\mathrm{s} 0$, $\mathrm{x}|\leq| \mathrm{Wf}(\mathrm{s} 0, \mathrm{x} 0) \mid$. The line connecting all the maximum modulus points in scale space $(\mathrm{s}, \mathrm{x})$ is called as modulus maxima line.

Definition 2 shows that the maximum modulus point of wavelet transform, namely, $(\mathrm{s} 0, \mathrm{x} 0)$ is strictly local maximum at both right and left neighborhoods of $\mathrm{x} 0$. The theorems about maximum modulus and the singularity of functions are followed.

Theorem 3: Given $\mathrm{n}$ is a strictly positive integer, $/$ is a wavelet with n-order vanishing moments, continuously differentiable to the power $\mathrm{n}$ and compact support, $\mathrm{f}(\mathrm{x}) \in \mathrm{L} 1([\mathrm{a}, \mathrm{b}])$, so

(1) If the scale $\mathrm{s} 0>0$ makes no local maximum point in $\forall \mathrm{s}<\mathrm{s} 0, \mathrm{x} \in(\mathrm{a}, \mathrm{b}),|\mathrm{Wf}(\mathrm{s}, \mathrm{x})|$, we will have $\forall \varepsilon>0$ and $\mathrm{a}<\mathrm{n}, \mathrm{f}(\mathrm{x})$ is the consistent Lipschitz exponent $\mathrm{a}$ in $(\mathrm{a}+\varepsilon, \mathrm{b}-\varepsilon)$.

(2) If $\mathcal{J}(x)$ is a n-order derivative of a certain smooth function, in $(a+\varepsilon, b-\varepsilon) f(x)$ will be the consistent Lipschitz exponent $\mathrm{n}$.

Theorem 3 has proven that if wavelet transform has no maximum modulus at a fine scale, the function will have no singularity of the point in any neighborhood. The theorem below explains the 
characters of a kind of isolated singularity points from the view of the maximum modulus of wavelet transform.

Theorem 4: Set the wavelet transform of function $f(x)$ is defined in $(a, b), x \in(a, b)$. Suppose that there is a scale $\mathrm{s} 0>0$ and a constant $\mathrm{C}$ to make the local maximum value of $\forall \mathrm{x} \in(\mathrm{a}, \mathrm{b})$ and $\mathrm{s}<\mathrm{s} 0$, $\mathrm{Wf}(\mathrm{s}, \mathrm{x})$ in cone

$$
\left|x-x_{0}\right| \leq C_{S}
$$

So

(1) For any $\mathrm{x} 1 \in(\mathrm{a}, \mathrm{b}), \mathrm{x} 1 \neq \mathrm{x} 0, \mathrm{f}(\mathrm{x})$ is the Lipschitz exponent $\mathrm{n}$ in a neighborhood of $\mathrm{x} 0$.

(2) $\mathrm{a}$ is a non-integer and a is less than $\mathrm{n} . \mathrm{f}(\mathrm{x})$ is the Lipschitz exponent a at the point of $\mathrm{x} 0$ and if and only if constant A can make each maximum modulus in the cone defined in Formula (13) have

$$
|W f(s, x)| \leq A s^{a}
$$

Formula (14) is equivalent to

$$
\log |W f(s, x)| \leq \log A+a \log s
$$

If the maximum of wavelet transform can meet the cone distribution required in Theorem 4, Formula (15) indicates that the singularity degree of $\mathrm{f}(\mathrm{x})$ at the point $\mathrm{x} 0$ is always the maximum slope of straight line above $\log |\mathrm{Wf}(\mathrm{s}, \mathrm{x})|$ at logarithmic scale. All maximum points are in the cone and the cone points to $\mathrm{x} 0$. It means that $\mathrm{f}(\mathrm{x})$ at all other points $\mathrm{x} \in(\mathrm{a}, \mathrm{b}), \mathrm{x} \neq \mathrm{x} 0$ is Lipschitz exponent $\mathrm{n}$.

Assume that the wavelet $\phi(\mathrm{x})$ has symmetry support $[-\mathrm{K}, \mathrm{K}]$, the point set in plane $(\mathrm{s}, \mathrm{x})$

$$
\left|x-x_{0}\right| \leq K S
$$

is the influenced cone of $\mathrm{x} 0$. Theorem 2 has demonstrated that in general, it is insufficient to simply test the decay rate of wavelet transform for the influenced cone of $\mathrm{x} 0$. To detect the decay properties below the cone is necessary due to the oscillation may produce singularity at the point $\mathrm{x} 0$. Form the following theorems, it can be known that if the oscillation is not intense, the singularity of $\mathrm{f}(\mathrm{x})$ at the point $\mathrm{x} 0$ is characterized by the condition of the wavelet transform along with any line in the cone smaller than the influenced cone.

Theorem 5: Set $x \in R,(a, b)$ is a neighborhood of $x 0, f(x) \in L 2(R)$, there is a scale $s 0>0$, and when $\mathrm{s}<\mathrm{s} 0$ and $\mathrm{x} \in(\mathrm{a}, \mathrm{b})$, the figures of wavelet transform $\mathrm{Wf}(\mathrm{s}, \mathrm{x})$ remain the same. And suppose that there is a constant $\mathrm{B}$ and $\varepsilon>0$ to make all $\mathrm{x} \in(\mathrm{a}, \mathrm{b})$ and $\mathrm{s}>0$, have

$$
|W f(s, x)| \leq B s^{\varepsilon}
$$

here $\mathrm{x}=\mathrm{X}(\mathrm{s})$ is a curve in scale space $(\mathrm{s}, \mathrm{x})$ meeting the condition

$$
\left|x_{0}-X(s)\right| \leq C
$$

Hereinto $\mathrm{C}<\mathrm{K}$. If there is a constant $\mathrm{A}$ for any $\mathrm{s}<\mathrm{s} 0$ can make the wavelet transform meet

$$
|W f(s, X(s))| \leq A s^{\gamma}, 0 \leq \gamma \leq n
$$

where for any $\mathrm{A}<\mathrm{C}, \mathrm{f}(\mathrm{x})$ is Lipschitz exponent a.

Theorem 5 can be utilized to compute Lipschitz exponent of some nonisolated singularity based on the maximum modulus of wavelet transform. The figures for detection of local modulus maxima are used to observe wavelet transform has constant figures or not in the neighborhood of $\mathrm{x} 0$. The 
Lipschitz exponent of $\mathrm{f}(\mathrm{x})$ at the point $\mathrm{x} 0$ is obtained by the decay of wavelet transform modulus along with the maxima line for any convergence at point $\mathrm{x} 0$ in the effected cone.

\section{Experimental analysis}

\subsection{Experimental platform}

In the experiment, reflection method was applied for ultrasonic test to clearly test the depth of defect and a probe with center frequency of $5 \mathrm{MHz}$ was used. Besides, the paper built a testing platform control as needed to perform the rotation control of silicon rod with a detection for every 1o rotation. Furthermore, the reflected wave of $120 \mathrm{o}$ was adopted to analyze during the experiment. Meanwhile, the researchers made an artificial aperture with $16 \mathrm{~mm}$ in diameter on the silicon rod with the diameter of $152.4 \mathrm{~mm}$ for more accurate data analysis. The sketch map has been shown in Fig. 4-1.

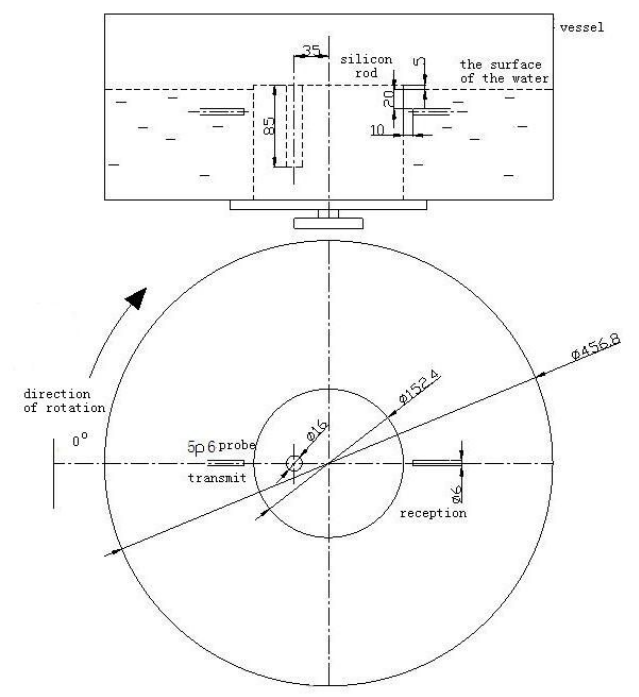

Fig.4-1 Sketch map of rotation bench

\subsection{Wavelet base selection [11]}

\subsubsection{The condition of wavelet base selection}

According to the experimental analysis above, choosing the proper wavelet base function can improve the detection efficiency greatly and it is really necessary. The wavelet base can be mainly selected from the following four aspects[9]:

(1) $\Psi(\mathrm{t})$ is compact support;

(2) $\Psi(\mathrm{t})$ is continuously differentiable;

(3) $\Psi(\mathrm{t})$ is n-order vanishing moment;

(4) $\Psi(\mathrm{t})$ is with symmetry.

They are the necessary conditions to solve the wavelet base of function singularity.

\subsubsection{The theoretical basis for selecting Gauss wavelet}

$$
\phi(t)=\frac{1}{\sqrt{\pi B_{m}}} \exp \left(\frac{-t^{2}}{B_{m}}\right) \exp \left(i 2 \pi f_{c} t\right)
$$


Formula (20) is a continuous Gaussian mother wavelet function, where $B_{m}$ is the bandwidth parameter of Gaussian mother wavelet; $f_{c}$ is its center frequency. As shown in Figure 4-2, it is the first-order Gaussian wavelet, while the second-order Gaussian wavelet has been illustrated in Figure 4-3. Since the most multi-scale edge detectors are smooth signals at different scales, and then using the first and second derivatives to detect the sharp change points, the edge detection can be performed by wavelet transform. In the case of smooth function is Gaussian function, the zero-crossing detection of the second derivative is equivalent to Marr edge detection, while the extremum detection of the first derivative is equivalent to Canny edge detection. Although they share some similarities, the extremum detection of the first derivative has prominent advantages. For instance, the inflection point is likely to be the maximum point or the minimum point of the absolute value of the first derivative, and the maximum reflects the sharp change while the minimum shows the slower change. Therefore, finding the maximum point is necessary to carry out the signal singularity detection. When the scale tends to zero, the decay speed of waveform depends on the Lipschitz exponent of the signal at the singular point. Thus, to find the extreme points of the first derivative can get more edge information than looking for the zero-crossing detection of the second derivative.

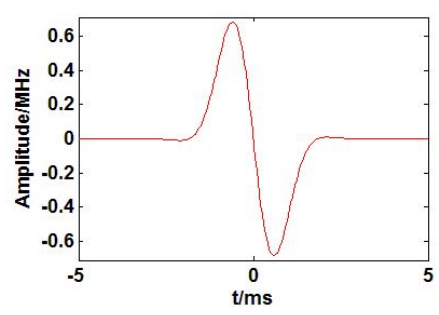

Fig.4-2 The first order Gaussian wavelet

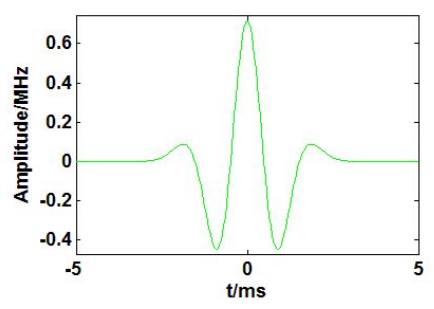

Fig.4-3 The second order Gaussian wavelet

In fact, in addition to the above advantages, Gaussian wavelet has four features of ultrasonic signal processing below.

1) On the basis of Heisenberg Uncertainty Principle[12], Gaussian wavelet has the best time-frequency resolution and the optimal time-frequency concentration;

2) Figures 4-2 and 4-3 show that Gaussian wavelet can match the ultrasonic pulse signal well;

3) From Formula (20), it can be seen that the phases of Gaussian wavelet $2 \pi f_{c} t$ is a liner.

4) The physical significance of the bandwidth of Gaussian wavelet $B_{m}$ and the center frequency $f_{c}$ is clear.

4.3 Experimental results and discussion

According to the appropriate wavelet is continuously differentiable with compact support, $\mathrm{N}$-order vanishing moments and symmetry, the paper has applied the Gaussian wavelet base to analyze the defects in the original signal (see in Fig. 4-2). Figure 4-3 has indicated the signals after wavelet transform of the defect wave; Fig. 4-4 is about the scale-location modulus distribution of the original flaw signals after wavelet transform, while Fig. 4-5 has suggested the distribution of the maximum modulus line obtained by algorithm. From Fig. 4-6, it can be known the logarithmic relationship between the maximum modulus and scale. 


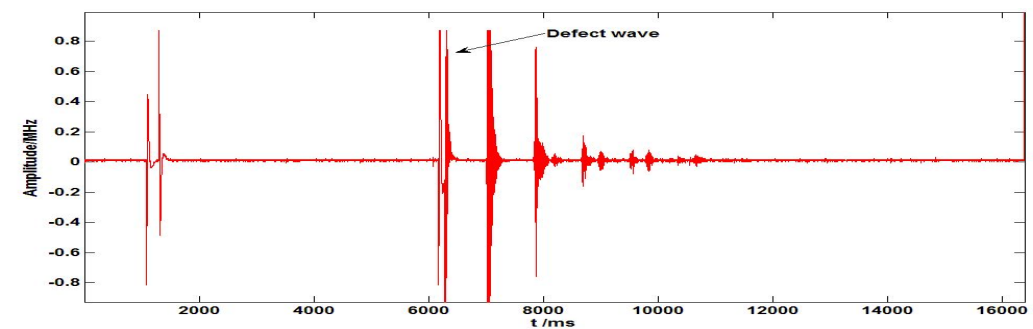

Fig.4-4 Original signals

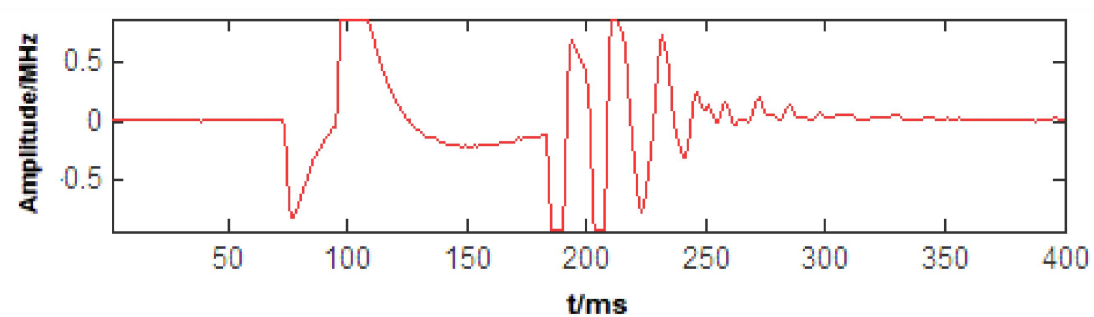

Fig 4-3 The signal of defect wave after wavelet transform

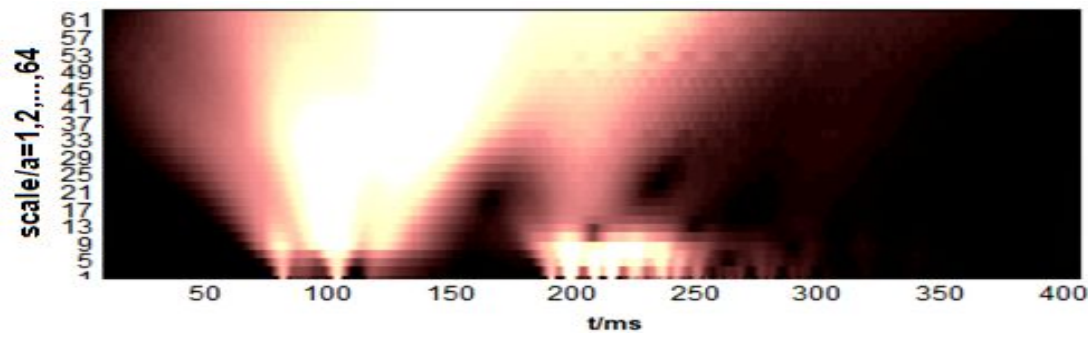

Fig. 4-4 Distribution of scale-position modulus value

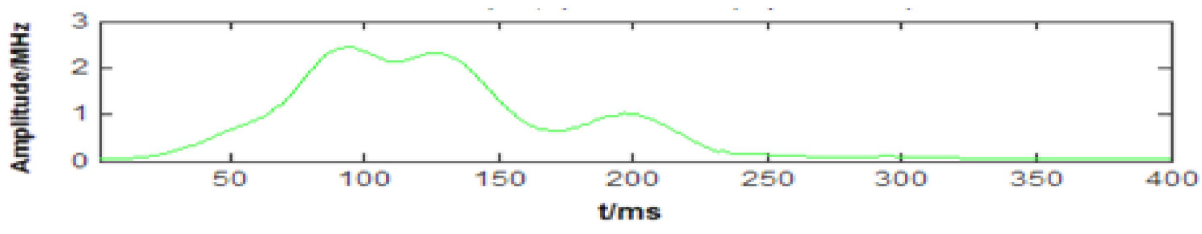

(a)Coefficients Line

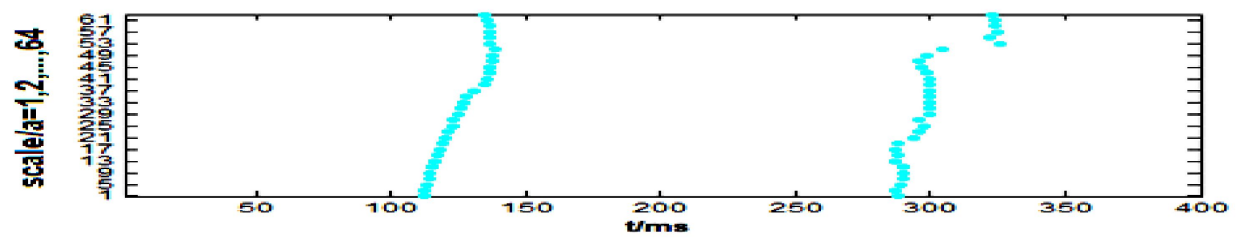

(b)Local Maxima Lines

Fig . 4-5 Local maximum modulus

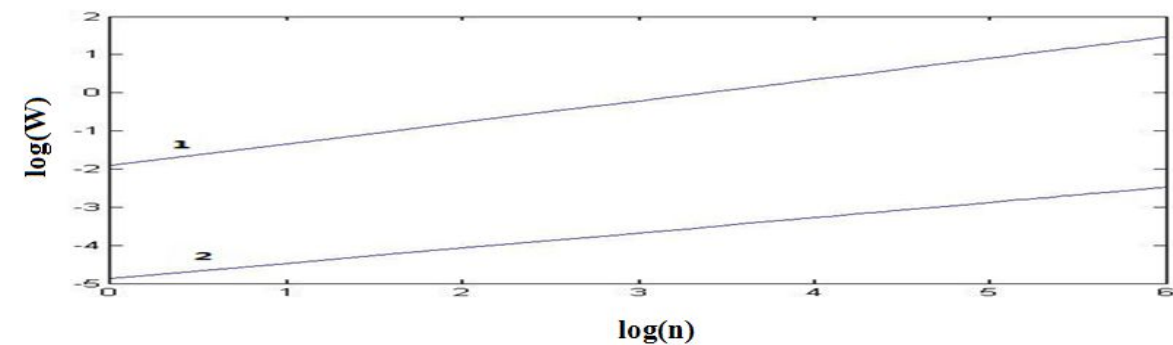


Fig. 4-6 The logarithmic relationship between the maximum modulus and scale

As shown in Fig 4-2, the points between original wave and a back reflection were used as a benchmark. The datum points can be regarded as the all points collected from the diameter of the silicon rod. The paper performed the continuous wavelet transform at the scale range of $1 \leq s \leq 64$. There are two obvious maximum modulus points: $n=106$ and $n=286$ (Fig. 4-5). In order to analyze the singularity property, a logarithmic relationship curve of the maximum modulus and scale is drawn (Fig. 4-6). In this figure, the slope of the line denotes the Lipschitz exponent of describing singularity, $\mathrm{a}=0.56$, while Curve 2 is the logarithmic relationship curve in the case of $n=286$, and the Lipschitz exponent is $\mathrm{a}=0.39$. Through analysis, it is likely to obtain that the two points represent the positions of the front and rear surfaces respectively. It usually takes about 33us from the original to a back reflection with 1753 points (Fig. 4-2). Besides, the propagation speed of ultrasonic wave in monocrystalline silicon is $\lambda=9408 \mathrm{~m} / \mathrm{s}$. Figure $4-5$ shows that there are 180 points of the front and rear surfaces of defect. The time interval between the front and rear surfaces of defect can be computed by the proportion relationship:

$$
t=\frac{33}{1735} \times 180 \cong 3.423 \mathrm{~s}
$$

Thus, the diameter of defect is:

$$
D=\frac{t \times \lambda}{2}=\frac{3.423 \times 10^{3} \times 9408}{1735} \cong 16.1 \mathrm{~mm}
$$

The actual diameter of defect is $16 \mathrm{~mm}$. The test positions were carried out by the circumference traversal testing bench. In order to further prove the validity of the test, the paper compared with the results of different testing positions through the rotation of the testing platform. Totally there were 359 different positions and all the defect diameters were computed. Table 1 has listed the data of four special positions and the data proved that the method using the singularity of wavelet is effective.

Table 1 The defect diameters at different testing positions

\begin{tabular}{|c|c|c|c|c|}
\hline $\begin{array}{c}\text { Angle } \\
(\mathrm{o})\end{array}$ & $\begin{array}{c}\text { Maximum } \\
\text { modulus points n1 }\end{array}$ & $\begin{array}{c}\text { Maximum } \\
\text { modulus points } \mathrm{n} 2\end{array}$ & Points number & $\begin{array}{l}\text { Defect diameters } \\
\mathrm{D}(\mathrm{mm})\end{array}$ \\
\hline 0 & 35 & 216 & 181 & 16.1 \\
\hline 90 & 72 & 253 & 181 & 16.1 \\
\hline 180 & 28 & 206 & 178 & 15.9 \\
\hline 270 & 23 & 206 & 183 & 16.3 \\
\hline
\end{tabular}

During the experiment, Daubechies (db) wavelet and ultrasonic signal also show favorable matching character. The property of the wavelet group is similar. And it has a better noise removal effect than the others. According to the selection principle of wavelet base, db wavelet transform was adopted for singularity detection to confirm the application of Gaussian wavelet in the monocrystalline silicon defect detection. Table 2 indicated the defect diameters computed by singularity detection through Gaussian and $\mathrm{db}$ wavelet for ten times.

Table 2 The detection results of Gaussian wavelet and $\mathrm{db}$ wavelet 


\begin{tabular}{|l|l|l|}
\hline NO. & Gauss wavelet $(\mathbf{m m})$ & DB wavelet $\quad(\mathbf{m m})$ \\
\hline 1 & 17.2 & 18.2 \\
\hline 2 & 16.1 & 15.6 \\
\hline 3 & 15.7 & 16.1 \\
\hline 4 & 16.8 & 17.5 \\
\hline 5 & 17.3 & 18.8 \\
\hline 6 & 16.9 & 18.4 \\
\hline 7 & 16.2 & 17.2 \\
\hline 8 & 16.3 & 17.2 \\
\hline 9 & 15.9 & 15.5 \\
\hline 10 & 17.1 & 17.6 \\
\hline
\end{tabular}

It is likely to see from Table 2, although the maximum modulus point has nothing to do with scale, in other words, due to the calculation error, the maximum modulus point has a certain disturbance. However, the detection results of Gaussian wavelet are better than those of $\mathrm{db}$ wavelet. The paper detected the root-mean-square error (RMSE) of monocrystalline silicon defect by 360 independent experiments. The RMSE of Gaussian wavelet and $\mathrm{db}$ wavelet are 0.6122 and 1.2195 respectively. Therefore, the detection results of Gaussian wavelet are more suitable.

The monocrystalline silicon is cylindrical, and the self-built circumference traversal testing bench was adopted in the experiment with a detection every 10 rotation. The single detection used preorder traversal algorithm to solve the quantization of the size of defects, but the testing data cannot reflect the location of the actual defects in the silicon. Therefore, all the data of $0 \mathrm{o} \sim 360 \mathrm{o}$ should be utilized to reflect the defects precisely. Figure 4-7 shows the reconstruction of monocrystalline silicon defect.

The discontinuity of monocrystalline silicon edge after reconstruction was induced by multiple overlapping of ultrasonic echo. In addition, with the rotation of monocrystalline silicon, the echo amplitude reduced when it was far from the defects so that its image was not clear. Moreover, the detection error may affect the reconstruction. Anyway, it is likely to know that the image can still reflect the three-dimension information of the defects with $16.6 \mathrm{~mm}$ in diameter. Compared with the actual artificial aperture, it remains the real size basically with a relative error of $3.75 \%$. In general, the result is desirable.

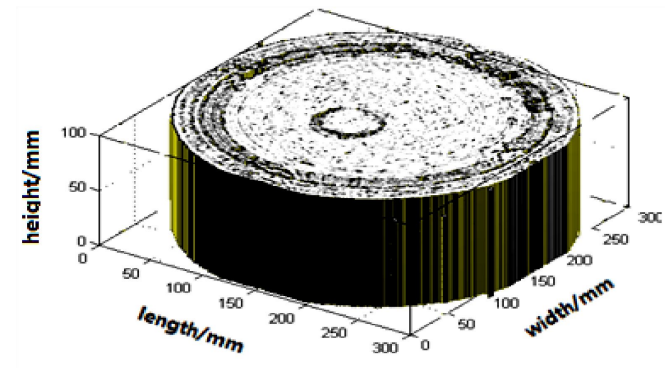

(a) opaque processing

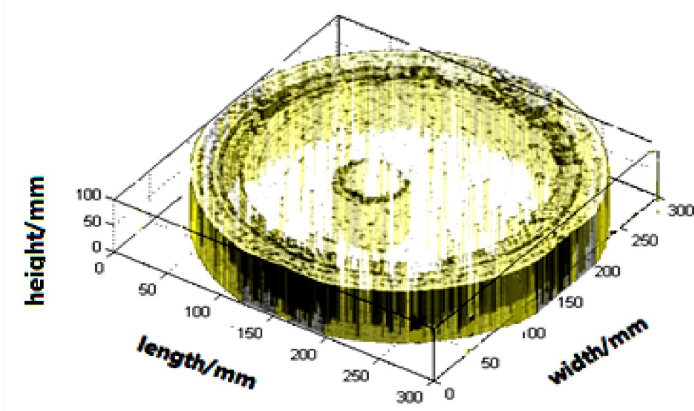

(b) transparent processing

Fig.4-7 The reconstruction of monocrystalline silicon defect

\section{Conclusion}

To improve the efficiency of monocrystalline silicon slicing, it is essential to precisely locate the defect in monocrystalline silicon in advance. First of all, based on the theoretical relationship between Lipschitz exponent proved by using Mallat and the local maximum modulus of wavelet, the paper solved the concrete values of Lipschitz exponent. By using the method into the analysis of 
the flaw signals of monocrystalline silicon, Gaussian wavelet is regarded as the mother wavelet to reduce noise in effect so as to accurately obtain the measured diameter of monocrystalline silicon defect. By doing so, a three-dimensional graph for showing internal defect of monocrystalline silicon can be reconstructed. And then the authors compared the actual values with the theoretical values of defect waves, as well as compared with the singularity of db wavelet as the mother wavelet. The experimental results have shown that this method has high sensitivity and strong ability of reducing noise. It can effectively improve the accuracy and speed of the detection for the diameter of wavelet defect, and is of great significance to locate and detect the internal defect diameter of monocrystalline silicon. Moreover, this method can be widely applied for the extraction of the eigenvalue of ultrasonic signal and fault detection.

It can be seen that this algorithm also shows some shortcomings, such as certain errors caused by the disturbance of maximum modulus and the delay or displacement at short distance led by ultrasonic signal at a large scale. The algorithm is expected to be improved in our future studies. For instance, the more effective nondestructive testing model of monocrystalline silicon can be further studied aiming at the transducers with different sizes, edge detection algorithm and defect type, as well as the improvement of reconstruction algorithm.

\section{Acknowledgments}

This work was financially supported by a grant from National Natural Science Foundation of China (No.51405382,No.51305346)

\section{Reference}

[1] Chen Jianzhong, Shi Yaowu, Shi Shu. Signal processing in ultrasonic testing of coarse grain materials. Nuclear Power Engineering, 2000, 21(22):183-187.

[2]Zhang Xiaofei, Wang Zuo, Zhou Youpeng. Noise processing in ultrasonic testing. Nondestructive Testing, 2002, 24(5): 200-202(229).

[3] Cvet kovic Z, VetterliM. Discrete-time wavelet extreme representation: Designand consistent Reconstruction. IEEE Trans Signal Processing, 1995, 43(3): 681-693.

[4] Berman Z, Baras J. Properties of the multiscale maxima and zero-crossing representation. IEEE Trans SP, 1993, 41(12): 3216.

[5] Cetin A, Ansari R. Signal recovery from wavelet trans form maxima. IEEE Trans SP, 1994, 42(1):194.

[6] Gustafsson MG, Stepinski T.Split spectrum algorithms rely on instant aneous phase informationage ometrical approach[J].IEEE Trans UFF C, 1993, 40(6):659-665.

[7] Zhou Zhijin, Wen Zhejun, Bu Yingyong.Application study of wavelet analysis on ultrasonic echo wave noise reduction.Chinese Journal of Scientific Instrument.2009, 30(20):237-241.

[8] Mallat S, Hwang Wenliang. Singularty detection and processing with wavelets. IEEE Trans. on IT, 1992, 38(2): 617-643.

[9] Eliane Martins Amanda, Rosa CA. A fault injection approach base on reflective programming. DSN. 2000, 2000, 1:407-416.

[10]Lian Ke, Wang Houjun, Long Bing. A novel method of measuring Lipschitz exponent based on wavelet transform modulus maxima.Acta Electronica Sinica. 2008, 36(1):106-110.

[11]Wu Zhi cheng, Wang Chongyang, Ren Aijun.Optimal selection of wavelet base functions for eliminating signal trend based on wavelet analysis. Transactions of Beijing Institute of Technology. 2013, 33(8): 811-814.

[12]Mallat S A Wavelet tour of signal processing. Bejing, China Machine Press, 2002, 182-201. 